\title{
BMJ Open Randomised controlled trial of a web- based low carbohydrate diet intervention for adults with type 2 diabetes: the T2Diet study protocol
}

\author{
Jedha Dening (D) , ${ }^{1}$ Elena S George, ${ }^{1}$ Kylie Ball, ${ }^{1}$ Mohammadreza Mohebbi, ${ }^{2}$ \\ Sheikh Mohammed Shariful Islam ${ }^{1}$
}

To cite: Dening J, George ES, Ball $\mathrm{K}$, et al. Randomised controlled trial of a webbased low carbohydrate diet intervention for adults with type 2 diabetes: the T2Diet study protocol. BMJ Open 2022;12:e054594. doi:10.1136/ bmjopen-2021-054594

- Prepublication history for this paper is available online. To view these files, please visit the journal online (http://dx.doi org/10.1136/bmjopen-2021 054594).

ESG, KB and SMSI contributed equally.

Received 17 June 2021 Accepted 17 January 2022

D Check for updates

(C) Author(s) (or their employer(s)) 2022. Re-use permitted under CC BY-NC. No commercial re-use. See rights and permissions. Published by BMJ.

${ }^{1}$ Institute for Physical Activity and Nutrition, School of Exercise and Nutrition Sciences, Deakin University, Burwood, Victoria, Australia

${ }^{2}$ Biostatistics Unit, Faculty of Health, Deakin University, Burwood, Victoria, Australia

Correspondence to

Jedha Dening;

deningje@deakin.edu.au

\section{ABSTRACT}

Introduction Type 2 diabetes (T2D) management frequently involves a multidisciplinary care team. However, standard care for patients with T2D is the central role of the general practice physician, and consists of routine appointments to monitor glycaemic status and overall health. Dietary modification is an essential component of T2D management. Evidence suggests that a low carbohydrate diet (LCD) provides better clinical outcomes for people with T2D compared with other diets. However, providing dietary support in face-to-face settings is challenged by issues of availability and accessibility. Provided in conjunction with standard care, digital interventions can help bridge this gap. The objective of this paper is to describe the protocol of a randomised controlled trial (RCT) of a web-based intervention that will evaluate the effectiveness of standard care plus webbased LCD intervention when compared with standard care only.

Methods and analysis In a two-arm parallel RCT, 100 adults with non-insulin-dependent T2D aged between 40 and 89 years will be randomised to either a theoretically informed 16-week automated web-based LCD intervention plus standard care or standard care only. LCD recommendations emphasise consuming nutrientdense whole foods and encourage a daily carbohydrate goal of 50-100 g, with an objective of achieving $10 \%$ to $<26 \%$ carbohydrates from total energy intake. Assessments will take place at baseline and 16 weeks. The primary outcome will be haemoglobin A1c. Additional data collected will include dietary intake, self-efficacy, weight and height, anti-diabetes medication and dosages, and diabetes-related comorbidities. Process evaluation will consist of a mixed-methods assessment of website engagement metrics, user experience and participants' perspectives.

Ethics and dissemination All study procedures have been approved by the Deakin University Human Research Ethics Committee (2020-349). Study findings will be disseminated widely through public, professional and academic presentation and publication.

Trial registration number Australian New Zealand Clinical Trials Registry (ACTRN12621000096853).
Strengths and limitations of this study

To the best of our knowledge, this is the first randomised controlled trial to evaluate the effectiveness of a web-based low carbohydrate diet $(10 \%$ to $<26 \%$ energy intake) intervention on glycaemic control in adults with type 2 diabetes.

- A key strength of the study is the randomised controlled design and robust outcome assessment using haemoglobin $\mathrm{A} 1 \mathrm{C}$ that will minimise bias and maximise the validity of the study findings.

- One limitation is no long-term follow-up, as this was not feasible for this study.

\section{INTRODUCTION}

The global burden of type 2 diabetes (T2D) was estimated at 462 million individuals in 2017. ${ }^{1}$ Due to metabolic changes, T2D results in high glycaemic status, frequently measured by haemoglobin A1c (HbA1c). The primary treatment goal is to assist people with T2D to achieve an HbAlc below 7.0\%. ${ }^{2}$ However, in 2020 , estimates indicated that $50 \%$ of adults with T2D had uncontrolled T2D, with HbA1c levels above the treatment goal. ${ }^{3}$ Uncontrolled T2D significantly contributes to the development of diabetes complications and mortality. ${ }^{3}$ Management of T2D frequently involves engagement of a multidisciplinary healthcare team to ensure that the needs of individuals are met comprehensively. However, the general practice physician (GP) plays the central role in providing standard care for T2D management. ${ }^{4}$ Standard care for patients with T2D consists of routine health checks with their GP to monitor glycaemic status, diabetes complications and overall health. ${ }^{2}{ }^{4}$ In addition, guidelines suggest that healthy behaviour should be routinely encouraged before or in conjunction with pharmacological treatment if necessary. ${ }^{24}$ 
Dietary modification plays an integral role in diabetes management, in improving glycaemic control and overall health. ${ }^{2}$ In terms of diet, a low fat, moderatehigh carbohydrate diet has traditionally been a common dietary recommendation provided to people with T2D. ${ }^{45}$ However, a growing body of evidence has demonstrated that low carbohydrate diets (LCDs), defined as $10 \%$ to $<26 \%$ carbohydrate of total energy intake,${ }^{67}$ may be more optimal for improving clinical outcomes in people with T2D. ${ }^{689}$ LCDs had previously been viewed as controversial. However, the growing body of evidence has prompted updates across international diabetes care guidelines, which have acknowledged LCDs as a safe and viable dietary option for people with T2D. ${ }^{2}{ }^{10-12}$ Systematic reviews and meta-analyses of LCDs in people with T2D have consistently demonstrated greater improvements in glycaemic control, increases in high-density lipoprotein (HDL) cholesterol, decreases in triglycerides, reduced medication requirements ${ }^{6891314}$ and potential for diabetes remission. ${ }^{9}$ In addition, significant improvements have been demonstrated in people with T2D provided with LCD recommendations through routine clinical care. ${ }^{15}$

LCD interventions for people with T2D have typically been delivered in face-to-face settings. ${ }^{8}$ However, people with T2D face substantial challenges in accessing dietary support due to limited availability, accessibility and cost barriers. ${ }^{2}{ }^{16-18}$ Provided in conjunction with standard care, web-based interventions can help bridge this gap, offering the potential for greater reach and accessibility, with the advantage of being convenient and on-demand to participants when required. ${ }^{19}$ Systematic reviews of web-based comprehensive self-management interventions in people with T2D have demonstrated favourable improvements in glycaemic control. ${ }^{20-22}$ Preliminary evidence suggested that web-based dietary interventions may be an effective way to support dietary change and improved glycaemic status in adults with T2D ${ }^{23}$ Furthermore, web-based interventions in people with T2D have shown promise as a cost-effective option, ${ }^{24}$ with the capacity to be widely implemented to support routine primary care. ${ }^{25}$ No randomised controlled trial (RCT) to date has assessed the effectiveness of an LCD intervention in individuals with T2D, delivered in a web-based setting.

The study protocol for an RCT of a web-based LCD programme for adults with T2D is presented here. The primary aim of this study is to determine the effectiveness of a web-based LCD intervention on glycaemic control in adults with T2D. We hypothesise that the web-based LCD intervention plus standard care will result in better glycaemic control-lower HbAlc levels-at 16 weeks compared with standard care alone in adults with T2D. Secondary aims are to assess changes in dietary intake, self-efficacy, weight and body mass index (BMI), antidiabetes medication and diabetes-related comorbidities; and to assess process outcomes related to user engagement and experience.

\section{METHODS}

\section{Study design}

The T2Diet study is a 16-week two-arm parallel RCT that aims to investigate the effectiveness of a web-based LCD intervention plus standard care versus standard care alone on glycaemic control in 100 Australian-based adults with T2D (figure 1). A period of 16 weeks was chosen as previous web-based dietary interventions demonstrated that significant improvements in glycaemic control could be achieved within this timeframe. ${ }^{23}$

Inclusion criteria will be adults aged $40-89$ years, the most highly affected demographic for T2D in Australia, ${ }^{26}$ with self-reported non-insulin-dependent T2D and selfreported HbA1c levels $\geq 7.0 \%$ within the previous 6 months; access to the Internet; an active email address; able to read and understand English; based in Australia; and willing and able to provide informed consent. All eligible participants with self-reported HbA1c levels $\geq 7.0 \%$ within the previous 6 months will be included, once baseline HbAlc measurements are conducted any reports returned as normal $\leq 5.6 \%^{2}$ will result in participants being excluded. Exclusion criteria will be people with type 1 diabetes, prediabetes or gestational diabetes; people with diagnosed renal or cardiovascular disease; people with a terminal disease or severe complications compromising the quality of life of the participant and their ability to participate according to the protocol; women who are pregnant or lactating; people who have undergone bariatric surgery; vegetarians or vegans; people currently on a weight loss programme or who have taken a weight loss programme within the past three months; people enrolled in other clinical studies; and people at risk of disordered eating, assessed during screening with the Eating Attitudes Test-26. ${ }^{27}{ }^{28}$ Participants identified with potential eating disorders will be referred to The Butterfly Foundation National Helpline. ${ }^{29}$ Informed consent will be obtained from eligible participants prior to entry into the study.

\section{Intervention}

The intervention is a theoretically informed 16-week automated web-based LCD behaviour change support programme. Existing website resources were provided for this study. Subsequently, four phases of inquiry were conducted with end-users (adults with T2D) to inform development of the new web-based dietary intervention. The weekly behaviour change modules adopt various behaviour change techniques ${ }^{30}$ and were constructed on a theoretical framework, consisting of: (1) self-efficacy theory ${ }^{31}$ — self-efficacy being a key determinant of self-care behaviours and glycaemic control in T2D $\mathrm{D}^{32-35}$; (2) positive message framing-using language that communicates benefits rather than scare tactics; ${ }^{36} 37$ and (3) principles of persuasive technology-using technology as a means of persuasively communicating intervention content. ${ }^{38}$

\section{Dietary recommendations}

Intervention participants will receive web-based recommendations to consume an ad libitum $\mathrm{LCD}^{13}$ and 


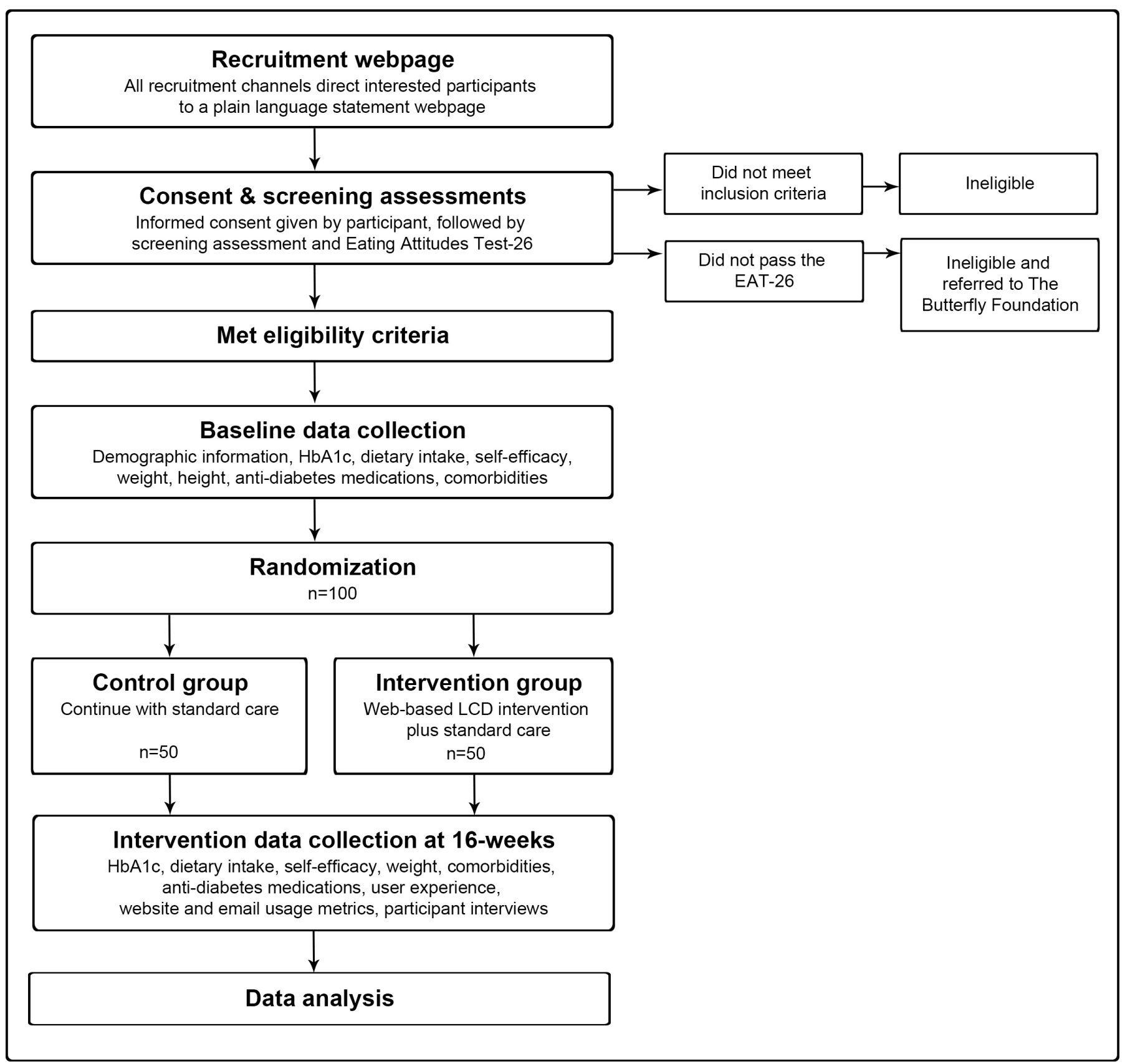

Figure 1 Study flow chart. EAT-26, Eating Attitudes Test-26; HbA1c, haemoglobin A1c; LCD, low carbohydrate diet.

encouraged to consume between 50 and $100 \mathrm{~g}$ carbohydrates per day. ${ }^{6}$ The overall goal is to achieve a low carbohydrate intake, defined as $10 \%$ to $<26 \%$ total energy intake. ${ }^{67}$ Web-based resources emphasise high consumption of non-starchy vegetables, adequate dietary fibre and selection of nutrient-dense sources of lower carbohydrate foods. ${ }^{2}$ Participants will be instructed to avoid or minimise high carbohydrate food and beverage sources such as added sugar, sugar-sweetened beverages, ${ }^{2} 539$ starchy foods ${ }^{1540}$ and discretionary foods. ${ }^{25}{ }^{59}$ There will be no specific prescription for other macronutrients. However, based on estimated energy intake for the demographic of this study ranging from 1600 calories/6694 kJ to 2400 calories $/ 10041 \mathrm{~kJ},{ }^{41}$ the protein and fat ranges are estimated to fall between 60-180 g (15\%-30\% estimated energy intake) and 80-200 g (45\%-75\% estimated energy intake), respectively. ${ }^{2}{ }^{42-44}$ Web-based recommendations encourage nutrient-dense sources of protein and fat, ${ }^{15} 40$ emphasising consumption of polyunsaturated and monounsaturated fat, ${ }^{2} 39$ and suggesting reduced-fat dairy may be preferred. ${ }^{45}$ To facilitate implementation, skills-based resources such as recipes, information on T2D, food and beverage choices, menu examples, eating out tips, menu planning and food preparation tips, an interactive planner and cooking demonstrations will be provided.

Web-based intervention delivery

Intervention participants will be provided with login details for the study website to access the weekly modules and on-demand resources. The weekly modules consist 
Week 2 Modules

므 T2Diet guidelines recap

므 Benefits of fibre

므 Understanding $T 2 D$

므 Healthy blood sugar

므 Recipe suggestions

므 Action step

Week 2. T2Diet Guidelines Recap (Video - 3:41 minutes)

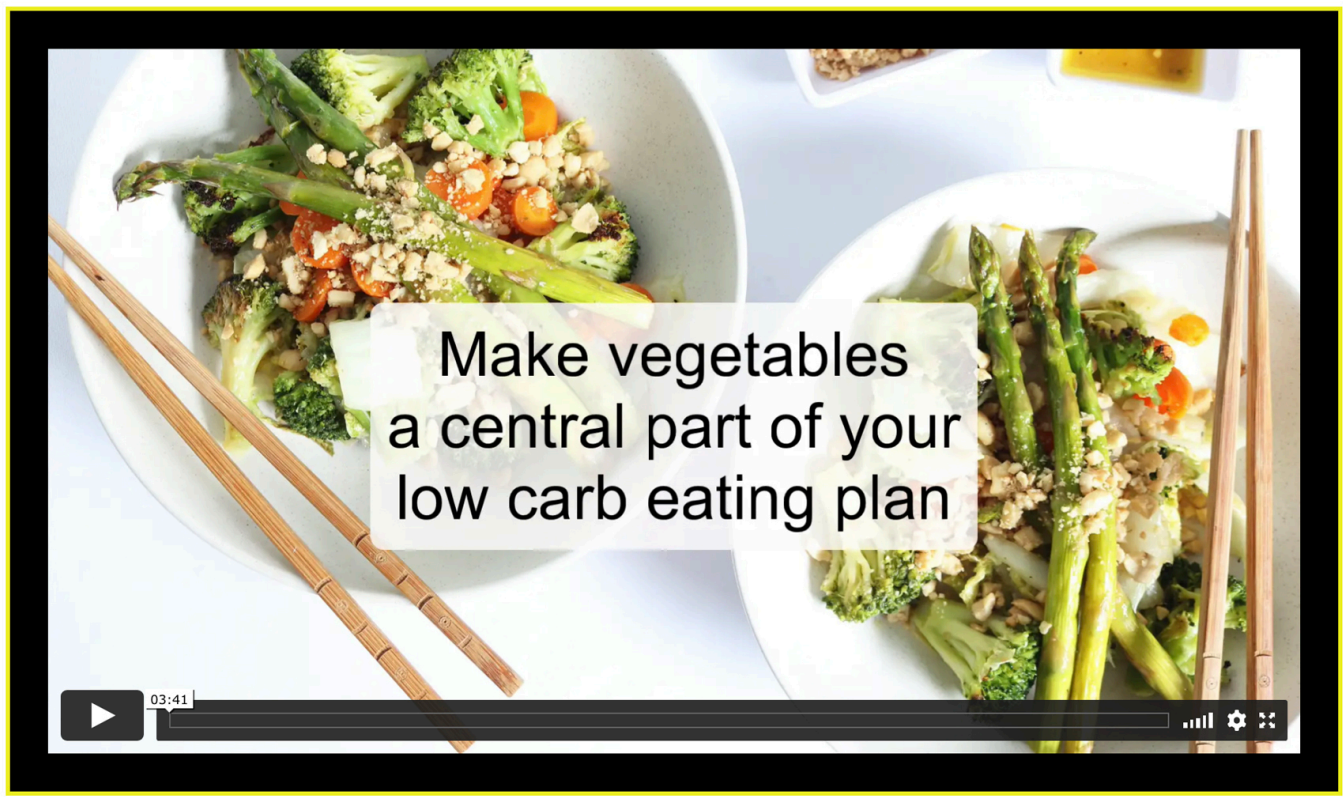

Brief overview

2 Essential Rules to Follow

Rule \#1: Eat Nutrient Dense Real Food

This means avoiding processed foods as much as possible and choosing fresh foods that contain more nutrients to help improve your health.

Rule \#2: Avoid or Minimise Sugar \& Starchy Foods

Sugar: many packaged food products contain high amounts of added sugar. Many beverages also contain a lot of sugar, including fruit juice which contains high amounts of naturally occurring sugar.

Starchy foods: potatoes, sweet potatoes, corn, bread, rice, pasta, noodles, cereals, flour and flour-based products.

Figure 2 Presentation format of the weekly behaviour change modules.

of short videos with brief overviews, links to further resources, recipe suggestions and action steps (figure 2). These delivery methods, particularly the use of video, have been shown to address various levels of literacy, ${ }^{46}$ enhance engagement ${ }^{47}$ and support health behaviour change. ${ }^{48}$ The weekly modules will be delivered sequentially, however, participants can continue to access any previous weeks' modules, along with accessing on-demand resources at any time. To prompt website usage and performance of behavioural actions, reminders will be sent to participants via email two times per week. ${ }^{46}$ It is estimated that participants will login to the website once per week.

\section{Adverse effects}

Intervention participants will be provided with education about and resources on how to manage potential adverse effects of carbohydrate reduction, such as constipation, headache and brain fog, halitosis, muscle cramps, tiredness and fatigue, hunger and cravings, and heart palpitations ${ }^{15}$; ; temporary hypoglycaemic-like symptoms; hypoglycaemia, defined as a blood glucose level $<70 \mathrm{mg} / \mathrm{dL} /<4.0 \mathrm{mmol} / \mathrm{L}$-emphasised for any participant taking sulfonylurea medications ${ }^{51}$; and to identify symptoms of ketoacidosis, for any participant taking sodium glucose cotransporter 2 inhibitor medications. $^{2} 51$ Participants will be able to report adverse effects via an online form. If participants request assistance, they will be directed to appropriate resources, and/or, advised to consult with their treating physician. Any adverse effects will be documented and reported with trial outcomes. 


\section{Intervention group follow-up}

Approximately three days after being provided with login details for the study website, intervention group participants will be followed up by email or phone to draw their attention to the potential adverse effects of carbohydrate reduction, cautions regarding medications and to encourage participants to discuss their participation in the study with their GP and healthcare team. Participants will be able to download a study information letter they can give to their GP or healthcare team.

\section{Standard care}

Participants in both groups will be advised to continue with their standard care, defined by the Royal Australian College of General Practitioners, ${ }^{4}$ as routine appointments with their physician to monitor glycaemic control, diabetes complications and other health parameters.

\section{Control condition}

The control group will be standard care, as defined above. Participants in the control group will be on a waitlist and provided with the opportunity to participate in the intervention after completing the study.

\section{Outcomes}

Primary and secondary outcomes will be measured at baseline and immediately post-intervention (16 weeks). An overview of study measures, data collection instruments and their timepoints is presented in table 1 . The primary outcome will be glycaemic control measured by the mean difference of change in HbAlc between intervention and control group from baseline to 16 weeks. For secondary outcomes, dietary intake data-food, beverages and dietary supplements, will be collected to assess participants' adherence to the recommended LCD. We will also explore improvements in the quality of the participants' diet such as changes in discretionary food intake and vegetable consumption, and changes to individuals' overall macronutrient and micronutrient intake and food groups. In addition, we will explore the association between adherence to diet and glycaemic control. Self-efficacy will be measured to assess whether participants' self-efficacy improves and whether self-efficacy predicts and promotes greater changes in glycaemic control. ${ }^{32-35}$ Weight and height will be collected to assess change in weight and BMI $\left(\mathrm{kg} / \mathrm{m}^{2}\right)$. Anti-diabetes medication and dosages and diabetes-related comorbidities ${ }^{4}$ will be collected to assess changes.

Table 1 Summary of outcome measures, data collection instruments and timepoint

\begin{tabular}{|c|c|c|c|}
\hline \multirow[b]{2}{*}{ Measures } & \multirow[b]{2}{*}{ Instrument } & \multicolumn{2}{|c|}{ Timepoint } \\
\hline & & Baseline & 16 weeks \\
\hline Demographic information & $\begin{array}{l}\text { Structured questionnaire, self-reported via an online request } \\
\text { form. }\end{array}$ & $x$ & \\
\hline \multicolumn{4}{|l|}{ Primary outcome } \\
\hline Haemoglobin A1c (\%) & $\begin{array}{l}\text { Collected and assessed using the Nutripath Integrative } \\
\text { Pathology Services } \mathrm{HbA} 1 \mathrm{c} \text { test. }\end{array}$ & $x$ & $x$ \\
\hline $\begin{array}{l}\text { Dietary intake: food, } \\
\text { beverage and dietary } \\
\text { supplements }\end{array}$ & $\begin{array}{l}\text { Assessed via self-reported 24-hour food recall using study- } \\
\text { specific online questionnaire, analysis using FoodWorks } \\
\text { professional nutrition software. }\end{array}$ & $x$ & $x$ \\
\hline Self-efficacy & $\begin{array}{l}\text { Self-reported via an online request form using the Diabetes } \\
\text { Management Self-Efficacy Scale-Australian version. }{ }^{66}\end{array}$ & $x$ & $\mathrm{x}$ \\
\hline \multicolumn{4}{|l|}{ Exploratory outcome } \\
\hline $\begin{array}{l}\text { Diabetes-related } \\
\text { comorbidities }\end{array}$ & Self-reported, via a study-specific online request form. & $X$ & $x$ \\
\hline \multicolumn{4}{|c|}{ Process outcomes (intervention group only) } \\
\hline Website utilisation & Website and email usage metrics. & & \\
\hline $\begin{array}{l}\text { User engagement and } \\
\text { experience }\end{array}$ & $\begin{array}{l}\text { Self-reported using the User Engagement Scale short form }{ }^{54} \\
\text { and Honeycomb Model, }{ }^{556} \text { via an online request form. }\end{array}$ & & $X$ \\
\hline $\begin{array}{l}\text { Participants' } \\
\text { experience }\end{array}$ & Semistructured phone interviews with up to 20 participants. & & $\mathrm{X}$ \\
\hline
\end{tabular}

BMI, body mass index. 


\section{Process evaluation}

Post-intervention, a mixed-methods approach ${ }^{52}$ will be used to explore website utilisation, user engagement and experience, in the intervention group only. Two forms of quantitative data will be collected: (1) website and email usage rates for frequency, intensity and duration metrics, ${ }^{53}$ collected for each week of the 16 -week intervention; and (2) a self-administered questionnaire using the User Engagement Scale short form ${ }^{54}$ and Honeycomb Model. ${ }^{5556}$ To collect qualitative data, a maximum of 20 participants ${ }^{57}$ will be invited to attend a semistructured phone interview. Process evaluation will be reported separately to the primary and secondary outcomes of this trial.

\section{Sample size}

A total of 100 participants (50 per group) will provide $80 \%$ power at type I error of 0.05 to detect a betweengroup difference of $0.5 \%$ on HbAlc (primary outcome). The sample size is based on the following assumptions: an SD of $0.9 \mathrm{HbA} 1 \mathrm{c},{ }^{58}$ a pre-post intervention correlation of $0.5^{60}$ and a dropout rate of $20 \% .^{6162}$ The effect size of $0.5 \%$ was chosen as it is considered a clinically meaningful HbA1c reduction. ${ }^{63}$ This may seem large for a relatively short intervention. However, it is not vastly different to previous LCD studies in people with T2D, where 6 month durations with smaller sample sizes demonstrated reductions in HbA1c of approximately $0.6 \% .^{585964}$ Previous LCD studies have indicated low dropout rates $(<10 \%)^{5964}$ and the average dropout across five web-based dietary interventions in people with T2D was approximately $22 \% .^{23}$ Therefore, a $20 \%$ dropout was considered reasonable for this study. The sample size calculation was conducted by an independent statistician using Stata's power twomeans command.

\section{Recruitment}

Enrolment of 100 participants will occur nationally across Australia using social media (primarily Facebook, Twitter and Linkedin); networking with colleagues and acquaintances; community publications, newsletters or radio; through diabetes clinics, community organisations, fitness centres or medical centres; snowballing; and if necessary, paid online advertising via Facebook. All recruitment channels will direct interested participants to a plain language statement webpage where participants can voluntarily provide their informed consent. After giving informed consent, participants are immediately redirected to the eligibility screening questionnaire. Eligible participants will then be required to complete all baseline measurements (table 1) before being randomised to their allocated group. Following intervention completion at 16 weeks, participants will be required to complete the same measurements, excluding demographic details and height (table 1). Recruitment started in February 2021 and is expected to be completed by December 2021 .

\section{Assignment of interventions}

Participants will be randomised in a 1:1 ratio to standard care (control group) or standard care plus web-based LCD intervention (intervention group) using block randomisation with varying random block sizes and stratified by age and gender. A computer-generated predetermined randomisation schedule will be produced and held offsite by an independent statistician, who will indicate the group allocation as eligible participants are recruited. The group allocation will be concealed from researchers and participants until all baseline measures have been collected and the independent statistician has conducted the randomisation. Post-intervention outcomes, except the primary outcome, will be assessed via participant self-report. Primary outcome assessment will be blinded as HbA1c samples are assessed by the pathology lab with no disclosure of group allocation. Data analysts will be blinded to group allocation.

\section{Data collection tools}

Aside from $\mathrm{HbAlc}$, all points of data collection will occur online through structured questionnaires with selfreported entries, for both groups. Demographic information such as age, gender, duration of T2D, family history of T2D, country of birth, employment status, education level, relationship status and smoking status will be collected at baseline for descriptive purposes.

HbAlc will be assessed by Nutripath Integrative Pathology Services. Participants will be sent the HbA1c microsample self-administered test. Participants unable to collect a sufficient self-administered sample will be sent the HbA1c pathology-assisted blood draw test. In both instances, the HbAlc test kits will be mailed to participants and back to the pathology service. HbAlc is the most common clinical biomarker used to assess glycaemic control in RCTs that include people with T2D. ${ }^{8}$

Dietary intake will be assessed using 24-hour food recall, a comprehensive self-report instrument that is considered highly robust. ${ }^{65}$ An online 24-hour food recall questionnaire will collect participants' self-report of foods, beverages and supplements consumed in the previous 24-hour period. Submissions will be reviewed and if necessary, participants will be contacted for additional detail. FoodWorks professional nutrition analysis software for Australia and New Zealand will be used.

Self-efficacy will be measured using the Diabetes Management Self-Efficacy Scale-Australian version, ${ }^{66}$ which has been validated for use in people with T2D in Australia. The scale contains 20 questions rated on a 10-point scale. Responses are then summed to present a single self-efficacy score. Higher scores indicate greater self-efficacy.

Self-reported weight and height, anti-diabetes medication and dosages and diabetes-related comorbidities will be collected. Online self-reported weight and height has been demonstrated to be a valid method. ${ }^{67}$ The checklist of comorbidities was drawn from the Royal Australian College of General Practitioners guidelines 
on general practice management of T2D. ${ }^{4}$ Reductions in anti-diabetes medication are commonly reported in LCD studies in people with T2D. ${ }^{813}{ }^{14}$ It has been noted that this reflects an underestimation in the overall benefits of LCDs. ${ }^{68}{ }^{69}$ Thus, consideration of the influence of medication requirements needs to be taken into account. The Medication Effect Score will be used to quantify and summarise the changes in anti-diabetes medication. ${ }^{70}$

\section{Participant retention and withdrawal}

To accommodate any loss, multiple imputations will be used to handle the missing data (either as primary or sensitivity analysis), using available data to minimise potential bias of estimated intervention effects due to nonrandom attrition. In addition, when measurement data are due/overdue, emails and/or text message reminders and phone calls to participants will be made. All participants completing this study will receive a \$A30 shopping voucher. Participants are free to withdraw from the study for any reason, up until data analysis commences.

\section{Data management and protection against bias}

Significant measures have been put in place to ensure robust data management and integrity and protect against bias. Primary outcome reports and self-reported data files will be downloaded in their wholly original unmodified form by the principal investigator and securely stored in a location inaccessible to other research team members. These original data files will not be modified. Copies of original data files will be provided to research team members as required. Data that require manual entry will be crosschecked against copies of the original data files by a second research team member. An independent statistician will be provided with a copy of the original or crosschecked data files to collate and clean the dataset in preparation for data analysis. Once complete, the coded, de-identified dataset will be securely stored by the principal investigator, as the lockdown dataset files, in a location inaccessible to other research team members. These lockdown dataset files will not be modified. Copies of the lockdown dataset will be shared with research team members as required. A dedicated study statistician will be provided with a copy of the lockdown dataset files to conduct the data analysis independently.

\section{Monitoring}

Overall study monitoring occurs via monthly meetings involving the research team members.

\section{Data availability statement}

Data will be available on reasonable request.

\section{Statistical methods}

All data will be imported into Stata for quantitative analysis. Baseline characteristics will be presented using descriptive statistics. The mean and SD, or median and range, will be used to describe continuous variables. Frequencies and/or percentages will be used to describe categorical variables. Intervention effects (ie, mean difference between intervention and control group) at 16 weeks will be evaluated by implementing an ANCOVA model for each outcome with the 16-week value of the outcome as the dependent variable, and treatment group and baseline outcome score as independent variables, and adjusting for stratification variables (age and gender). Multiple imputation techniques with missing at random assumption will be used to impute missing data due to dropouts or withdrawals to comply with the intention-totreat approach. Sensitivity analysis will be performed to evaluate missing at random assumption for missing observation pattern. Subgroup analysis will be conducted with the duration of diabetes and gender. For the duration of diabetes, a median split will be used to define subgroups. $P$ value 0.05 will be used as the level of significance for the primary outcome and all secondary outcomes. Cohen's D effect size will be calculated and reported. For all continuous outcomes, data will be explored for deviation from the normal distribution assumptions. If necessary, a transformation of data (eg, log transformation) or a nonparametric approach may be considered.

\section{Patient and public involvement}

Participants (adults with T2D) were engaged in four iterative phases of user-centred inquiry involving group discussions, which informed the development of the new web-based dietary intervention. Intervention participants will be involved in feedback during process evaluation.

\section{ETHICS AND DISSEMINATION}

All study procedures have been approved by the Deakin University Human Research Ethics Committee (2020349). Any protocol amendments will be submitted for approval to the ethics committee prior to implementation and communicated via an update of the Australian and New Zealand Clinical Trial Registry.

Key audiences this research may benefit include the general public, researchers, clinicians, policymakers and healthcare organisations. ${ }^{71-73}$ Various methods may be used to disseminate the findings, including peer-reviewed publication, presentations, consumer and professional publication and social media. ${ }^{73}$ Participants involved in the study will be sent a summary report of the study's main outcomes via email. In any dissemination of research findings, participants' identities will remain confidential.

\section{Limitations and strengths}

One limitation is that this study will not collect biomarkers related to cardiometabolic risk, which was beyond the scope of this trial. While more research is needed in this area, the overall evidence suggests that LCDs may be associated with cardiovascular benefits, as commonly a reduction in triglycerides and an increase in HDL cholesterol are observed. ${ }^{68136869}$ For low-density lipoprotein (LDL) cholesterol, the evidence remains unclear due to mixed reports. ${ }^{975-77}$ In addition, blood pressure can be influenced by LCDs. ${ }^{78}$ Given this web-based dietary 
intervention will be provided in conjunction with standard care, biomarkers such as lipid profiles and blood pressure would continue to be routinely monitored by the participants GP or healthcare team. The short duration of this trial is also a potential limitation. However, the duration was justified based on previous web-based dietary interventions, ${ }^{23}$ and given this will be the first RCT of a web-based LCD intervention, determining effectiveness prior to allocating additional time and resources will be important. Another potential limitation is the study will not measure other lifestyle-related factors such as physical activity or psychological well-being. ${ }^{2}$ The intervention was not designed to influence these outcomes, and any differences should be adequately addressed through random distribution in an RCT design. In addition, improvements related to hunger and satiety have been previously noted in LCD studies, ${ }^{68} 69$ though will not be collected for this trial. This study also has significant strengths. While only one primary biomarker will be included, it will enable this research to be conducted remotely. This makes the study highly feasible during COVID-19 when restrictions of movement and face-toface contact can be limited. In addition, remote delivery will increase the capacity to include participants from wide geographical locations, which will be of benefit given support for people with T2D in rural and remote areas is less accessible. ${ }^{79}$ Furthermore, the RCT design, allocation concealment and blinding are key strengths that will minimise bias and maximise the validity of the study findings.

\section{DISCUSSION}

This study will conduct an RCT of standard care alone vs standard care plus web-based LCD intervention in adults with T2D, with the primary intervention objective of improving glycaemic control. To meet the needs and context of end-users who will participate in the study, user-centred principles and involvement of end-users in numerous rounds of feedback and iterative development were employed. The weekly behaviour change modules apply various behaviour change techniques and were constructed on a theoretical framework to help strengthen communication of the intervention, address literacy levels and maintain engagement. In addition, the email reminder notifications aim to boost website usage and motivation to participate.

This study will be the first RCT of an LCD intervention for adults with T2D, delivered in a web-based setting. The findings will contribute valuable insights into whether an LCD is effective when delivered in a web-based environment; and whether such an intervention could be considered to support T2D management more broadly. Further, this study will contribute new knowledge to inform future digitally delivered dietary interventions that could be used to reach a greater number of people with T2D and other health conditions across Australia and internationally.
Twitter Jedha Dening @jedhadening, Elena S George @elenas_george, Kylie Ball @KylieBall3 and Sheikh Mohammed Shariful Islam @drsislam

Acknowledgements We thank Dr Gavin Abbott and Professor Ralph Maddison from Deakin University Institute of Physical Activity and Nutrition for their contributions.

Contributors JD conceived and designed the study and wrote the manuscript. SMSI, ESG, KB made critical contributions to the study design and provided critical input and revisions to the manuscript. MM contributed to the study design and provided critical revisions of the manuscript. All authors provided final approval for submission of the manuscript.

Funding This work will be supported by allocated annual $\mathrm{PhD}$ student funds from Deakin University Institute of Physical Activity and Nutrition.

Competing interests JD is co-owner of Diabetes Meal Plans, a web-based nutrition support service for people with type 2 diabetes and pre-diabetes, who licensed the web-platform for this study.

Patient and public involvement Patients and/or the public were involved in the design, or conduct, or reporting, or dissemination plans of this research. Refer to the Methods section for further details.

Patient consent for publication Not applicable.

Provenance and peer review Not commissioned; externally peer reviewed.

Open access This is an open access article distributed in accordance with the Creative Commons Attribution Non Commercial (CC BY-NC 4.0) license, which permits others to distribute, remix, adapt, build upon this work non-commercially, and license their derivative works on different terms, provided the original work is properly cited, appropriate credit is given, any changes made indicated, and the use is non-commercial. See: http://creativecommons.org/licenses/by-nc/4.0/.

ORCID iD

Jedha Dening http://orcid.org/0000-0003-0072-460X

\section{REFERENCES}

1 Khan MAB, Hashim MJ, King JK, et al. Epidemiology of Type 2 Diabetes - Global Burden of Disease and Forecasted Trends. $J$ Epidemiol Glob Health 2020;10:107-11.

2 American Diabetes Association. Standards of medical care in Diabetes-2021. Diabetes Care 2021;44:S1-232 https://www. diabetes.org/newsroom/press-releases/2020/ADA-releases-2021standards-of-medical-care-in-diabetes

3 Centers for Disease Control and Prevention. Risk factors for diabetes-related complications. 2020 June 30, 2020. Available: https://www.cdc.gov/diabetes/data/statistics-report/riskscomplications.html [Accessed 22 Mar 2021].

4 The Royal Australian College of General Practitioners. Management of type 2 diabetes: a Handbook for general practice, 2020.

5 Diabetes Australia. What should I eat? 2020. Available: https://www. diabetesaustralia.com.au/food-activity/eating-well/what-should-i-eat/ [Accessed 28 Apr 2021].

6 McArdle PD, Greenfield SM, Rilstone SK, et al. Carbohydrate restriction for glycaemic control in type 2 diabetes: a systematic review and meta-analysis. Diabet Med 2019;36:335-48.

7 Dening J, Islam SMS. Defining a low carbohydrate diet: proposal for a standardized consensus of carbohydrate intake (Carb-Cal model). Diabetes Res Clin Pract 2020;166:108284.

8 Huntriss R, Campbell M, Bedwell C. The interpretation and effect of a low-carbohydrate diet in the management of type 2 diabetes: a systematic review and meta-analysis of randomised controlled trials. Eur J Clin Nutr 2018;72:311-25.

9 Goldenberg JZ, Day A, Brinkworth GD, et al. Efficacy and safety of low and very low carbohydrate diets for type 2 diabetes remission: systematic review and meta-analysis of published and unpublished randomized trial data. BMJ 2021;372:m4743.

10 Diabetes Australia. Position statement: low carbohydrate eating for people with diabetes, 2018. https://www.diabetesaustralia.com.au/ wp-content/uploads/Diabetes-Australia-Position-Statement-LowCarb-Eating.pdf

11 Evert AB, Dennison M, Gardner CD, et al. Nutrition therapy for adults with diabetes or prediabetes: a consensus report. Diabetes Care 2019;42:731-54.

12 Davies MJ, D'Alessio DA, Fradkin J, et al. Management of hyperglycemia in type 2 diabetes, 2018. A consensus report 
by the American diabetes association (ADA) and the European association for the study of diabetes (EASD). Diabetes Care 2018;41:2669-701.

13 Sainsbury E, Kizirian NV, Partridge SR, et al. Effect of dietary carbohydrate restriction on glycemic control in adults with diabetes: a systematic review and meta-analysis. Diabetes Res Clin Pract 2018;139:239-52.

14 Snorgaard O, Poulsen GM, Andersen HK, et al. Systematic review and meta-analysis of dietary carbohydrate restriction in patients with type 2 diabetes. BMJ Open Diabetes Res Care 2017;5:e000354.

15 Unwin D, Khalid AA, Unwin J, et al. Insights from a general practice service evaluation supporting a lower carbohydrate diet in patients with type 2 diabetes mellitus and prediabetes: a secondary analysis of routine clinic data including $\mathrm{HbA} 1 \mathrm{c}$, weight and prescribing over 6 years. BMJ Nutr Prev Health 2020;3:285-94.

16 Australian Diabetes Educators Association. Workforce in diabetes education, 2019. Available: https://members.adea.com.au/resources2/workforce-in-diabetes-education/ [Accessed 19 Aug 2019].

17 Kennedy M, Dunning T. Diabetes education: essential but underfunded in Australia. Diabetes \& Primary Care Australia 2017;2:10-14.

18 Odgers-Jewell K, Isenring EA, Thomas R, et al. Group-Based education for patients with type 2 diabetes: a survey of Australian dietitians. Aust J Prim Health 2017;23:364-72.

19 Karekla M, Kasinopoulos O, Neto DD, et al. Best practices and recommendations for digital interventions to improve engagement and adherence in chronic illness sufferers. Eur Psychol 2019;24:49-67.

20 Cotter AP, Durant N, Agne AA, et al. Internet interventions to support lifestyle modification for diabetes management: a systematic review of the evidence. J Diabetes Complications 2014;28:243-51.

21 Pereira K, Phillips B, Johnson C, et al. Internet delivered diabetes self-management education: a review. Diabetes Technol Ther 2015;17:55-63.

22 Kebede MM, Zeeb H, Peters M, et al. Effectiveness of digital interventions for improving glycemic control in persons with poorly controlled type 2 diabetes: a systematic review, meta-analysis, and meta-regression analysis. Diabetes Technol Ther 2018;20:767-82.

23 Dening J, Islam SMS, George E, et al. Web-Based Interventions for Dietary Behavior in Adults With Type 2 Diabetes: Systematic Review of Randomized Controlled Trials. J Med Internet Res 2020;22:e16437.

24 Murray E, Sweeting M, Dack C, et al. Web-Based self-management support for people with type 2 diabetes (HeLP-Diabetes): randomised controlled trial in English primary care. BMJ Open 2017;7:e016009.

25 Ross J, Stevenson F, Dack C, et al. Developing an implementation strategy for a digital health intervention: an example in routine healthcare. BMC Health Serv Res 2018;18:794.

26 National Diabetes Service Scheme. Diabetes data snapshots: Type 2 diabetes - March 2021, 2021. Available: https://www.ndss.com. au/about-the-ndss/diabetes-facts-and-figures/diabetes-datasnapshots/ [Accessed 28 Apr 2021].

27 Young-Hyman DL, Davis CL. Disordered eating behavior in individuals with diabetes: importance of context, evaluation, and classification. Diabetes Care 2010;33:683-9.

28 Garner DM, Olmsted MP, Bohr Y, et al. The eating attitudes test: psychometric features and clinical correlates. Psychol Med 1982;12:871-8.

29 The Butterfly Foundation. Talking helps. butterfly national Helpline, 2021. Available: https://butterfly.org.au/Get\%20Support/Helpline/

30 Michie S, Richardson M, Johnston M, et al. The behavior change technique taxonomy (V1) of 93 hierarchically clustered techniques: building an international consensus for the reporting of behavior change interventions. Ann Behav Med 2013;46:81-95.

31 Bandura A. Self-Efficacy: the exercise of control. 9 edn. New York: W.H Freeman and Company, 1997.

32 Indelicato L, Dauriz M, Santi L, et al. Psychological distress, self-efficacy and glycemic control in type 2 diabetes. Nutr Metab Cardiovasc Dis 2017:27:300-6.

33 Brown SA, García AA, Brown A, et al. Biobehavioral determinants of glycemic control in type 2 diabetes: a systematic review and metaanalysis. Patient Educ Couns 2016;99:1558-67.

34 Ouyang C-M, Dwyer JT, Jacques PF, et al. Determinants of dietary self-care behaviours among Taiwanese patients with type 2 diabetes. Asia Pac J Clin Nutr 2015;24:430-7.

35 Strychar I, Elisha B, Schmitz N. Type 2 diabetes self-management: role of diet self-efficacy. Can J Diabetes 2012;36:337-44.

36 Paragas ED, Barcelo TI. Effects of message-framed informational videos on diabetes management knowledge and self-efficacy. Int $J$ Nurs Pract 2019;25:e12737.
37 Gans KM, Risica PM, Dulin-Keita A, et al. Innovative video tailoring for dietary change: final results of the good for you! cluster randomized trial. Int J Behav Nutr Phys Act 2015;12:130.

38 Kelders SM, Kok RN, Ossebaard HC, et al. Persuasive system design does matter: a systematic review of adherence to web-based interventions. J Med Internet Res 2012;14:e152.

39 National Health and Medical Research Council. Australian dietary guidelines. Canberra, 2013.

40 Turton J, Brinkworth GD, Field R, et al. An evidence-based approach to developing low-carbohydrate diets for type 2 diabetes management: a systematic review of interventions and methods. Diabetes Obes Metab 2019;21:2513-25.

41 US Department of Agriculture. 2015-2020 dietary guidelines for Americans, 2015. Available: https://health.gov/our-work/nutritionphysical-activity/dietary-guidelines/previous-dietary-guidelines/ 2015

42 Sucher S, Markova M, Hornemann S, et al. Comparison of the effects of diets high in animal or plant protein on metabolic and cardiovascular markers in type 2 diabetes: a randomized clinical trial. Diabetes Obes Metab 2017:19:944-52.

43 Gannon MC, Nuttall FQ. Effect of a high-protein, low-carbohydrate diet on blood glucose control in people with type 2 diabetes. Diabetes 2004;53:2375-82.

44 Pedersen E, Jesudason DR, Clifton PM. High protein weight loss diets in obese subjects with type 2 diabetes mellitus. Nutr Metab Cardiovasc Dis 2014;24:554-62

45 Heart Foundation. Dairy \& Heart Healthy Eating, 2019. Available: https://www.heartfoundation.org.au/getmedia/b7f8c612-c1eb-4139b4b1-898d8302d234/Nutrition_Position_Statement_-_DAIRY.pdf [Accessed 28 Apr 2021].

46 Pal K, Dack C, Ross J, et al. Digital Health Interventions for Adults With Type 2 Diabetes: Qualitative Study of Patient Perspectives on Diabetes Self-Management Education and Support. J Med Internet Res 2018;20:e40.

47 Abu Abed M, Himmel W, Vormfelde S, et al. Video-Assisted patient education to modify behavior: a systematic review. Patient Educ Couns 2014;97:16-22.

48 Desroches S, Lapointe A, Ratté S, et al. Interventions to enhance adherence to dietary advice for preventing and managing chronic diseases in adults. Cochrane Database Syst Rev 2013:CD008722.

49 LeRouge C, Wickramasinghe N. A review of user-centered design for diabetes-related consumer health informatics technologies. $J$ Diabetes Sci Technol 2013;7:1039-56.

50 Churuangsuk C, Lean MEJ, Combet E. Lower carbohydrate and higher fat intakes are associated with higher hemoglobin A1c: findings from the UK national diet and nutrition survey 2008-2016. Eur J Nutr 2020;59:2771-82.

51 Murdoch C, Unwin D, Cavan D, et al. Adapting diabetes medication for low carbohydrate management of type 2 diabetes: a practical guide. Br J Gen Pract 2019;69:360-1.

52 Creswell J. A Concise Introduction to Mixed Methods Research. California: Sage Publications, 2015.

53 Short CE, DeSmet A, Woods C. Measuring engagement in eHealth and mHealth behavior change interventions: viewpoint of methodologies 2018

54 O'Brien HL, Cairns P, Hall M. A practical approach to measuring user engagement with the refined user engagement scale (UES) and new UES short form. Int J Hum Comput Stud 2018;112:10.1016/j.ijhcs.2018.01.004:28-39.

55 Morville P. User experience honeycomb, 2016. Available: https:// intertwingled.org/user-experience-honeycomb/ [Accessed 20 Aug 2019].

56 University of Waterloo. User experience design for learning (UXDL), 2021. Available: https://cms.cel.uwaterloo.ca/honeycomb/index.aspx [Accessed 28 Apr 2021].

57 Braun V, Clarke V. To saturate or not to saturate? Questioning data saturation as a useful concept for thematic analysis and sample-size rationales. Qual Res Sport Exerc Health 2021;13:201-16.

58 Yamada Y, Uchida J, Izumi H, et al. A non-calorie-restricted lowcarbohydrate diet is effective as an alternative therapy for patients with type 2 diabetes. Intern Med 2014;53:13-19.

59 Jonasson L, Guldbrand H, Lundberg AK, et al. Advice to follow a low-carbohydrate diet has a favourable impact on low-grade inflammation in type 2 diabetes compared with advice to follow a low-fat diet. Ann Med 2014;46:182-7.

60 Clifton PM, Coles LT, Galbraith CE. Low carbohydrate diets in type 2 Diabetes - A translational study. J Diabetes Mellitus 2016;06:10.4236/jdm.2016.62016:152-7.

61 Jahangiry LS, Montazeri D, Najafi A. Adherence and attrition in a web-based lifestyle intervention for people with metabolic syndrome. Iranian J Publ Health 2014;43:1248-58. 
62 Ramadas A, Chan CKY, Oldenburg B, et al. Randomised-controlled trial of a web-based dietary intervention for patients with type 2 diabetes: changes in health cognitions and glycemic control. BMC Public Health 2018;18:716.

63 Heintjes E, Penning-van Beest FJA, Parasuraman SV, et al. PCV32 population attributable risk (PAR) of macrovascular events associated with $\mathrm{HbA1c}$, blood pressure or weight in patients with type 2 diabetes mellitus: evidence from a Dutch cohort. Value in Health 2011;14:A370-510.

64 Sato J, Kanazawa A, Makita S, et al. A randomized controlled trial of $130 \mathrm{~g} /$ day low-carbohydrate diet in type 2 diabetes with poor glycemic control. Clin Nutr 2017;36:992-1000.

65 National Cancer Institute. Dietary assessment primer, 2020. Available: https://dietassessmentprimer.cancer.gov/

66 McDowell J, Courtney M, Edwards $\mathrm{H}$, et al. Validation of the Australian/English version of the diabetes management self-efficacy scale. Int J Nurs Pract 2005;11:177-84.

67 Pursey K, Burrows TL, Stanwell P, et al. How accurate is web-based self-reported height, weight, and body mass index in young adults? J Med Internet Res 2014;16:e4.

68 Kelly T, Unwin D, Finucane F. Low-Carbohydrate diets in the management of obesity and type 2 diabetes: a review from clinicians using the approach in practice. Int J Environ Res Public Health 2020;17. doi:10.3390/ijerph17072557. [Epub ahead of print: $08 \mathrm{Apr}$ 2020].

69 Wheatley SD, Deakin TA, Arjomandkhah NC, et al. Low carbohydrate dietary approaches for people with type 2 Diabetes-A narrative review. Front Nutr 2021;8:687658.

70 Alexopoulos A-S, Yancy WS, Edelman D, et al. Clinical associations of an updated medication effect score for measuring diabetes treatment intensity. Chronic IIIn 2021;17:451-62.

71 National Health and Medical Research Council. The Australian code for the responsible conduct of research. Canberra, 2018.
72 National Health and Medical Research Council. Publication and dissemination of research: a guide supporting the Australian code for the responsible conduct of research. Canberra, 2020.

73 Agency of Healthcare Research and Quality. Communication and Dissemination Strategies To Facilitate the Use of Health and Health Care Evidence, 2012. Available: https://effectivehealthcare.ahrq.gov/ sites/default/files/pdf/medical-evidence-communication_researchprotocol.pdf

74 Ross-Hellauer T, Tennant JP, Banelytè V, et al. Ten simple rules for innovative dissemination of research. PLoS Comput Biol 2020;16:e1007704

75 Gjuladin-Hellon T, Davies IG, Penson P, et al. Effects of carbohydraterestricted diets on low-density lipoprotein cholesterol levels in overweight and obese adults: a systematic review and meta-analysis. Nutr Rev 2019;77:161-80.

76 Bhanpuri NH, Hallberg SJ, Williams PT, et al. Cardiovascular disease risk factor responses to a type 2 diabetes care model including nutritional ketosis induced by sustained carbohydrate restriction at 1 year: an open label, non-randomized, controlled study. Cardiovasc Diabetol 2018;17:56.

77 Mansoor N, Vinknes KJ, Veierød MB, et al. Effects of lowcarbohydrate diets $\mathrm{V}$. low-fat diets on body weight and cardiovascular risk factors: a meta-analysis of randomised controlled trials. Br J Nutr 2016;115:466-79.

78 Unwin DJ, Tobin SD, Murray SW, et al. Substantial and sustained improvements in blood pressure, weight and lipid profiles from a carbohydrate restricted diet: an observational study of insulin resistant patients in primary care. Int J Environ Res Public Health 2019;16. doi:10.3390/ijerph16152680. [Epub ahead of print: 26 Jul 2019].

79 National Diabetes Service Scheme. Living in rural and remote areas, 2021. Available: https://www.ndss.com.au/living-with-diabetes/ about-you/people-with-diabetes-living-in-rural-and-remote-areas/ [Accessed 06 Sep 2021]. 